\title{
Secured Way of Parking and Trackiing Vehicles using XMPP Protocol
}

\author{
Francis Brindha A, Sandhya Devi RS
}

\begin{abstract}
Nowadays there is a mass increase in numbers of vehicles in daily usage. And also there is an increase in problems regarding its parking and management also. Parking the vehicles in situations like underground parking in malls or large area parking and getting back the vehicles from that parked place is a tedious task and consumes lot of time. It needs some safety for our vehicles also. This paper proposed the module where the information regarding the free slots in parking area while parking and location of the vehicles while retrieving can be show to the user to make the process easy. And this information sharing will be in secured way. With the help of sensors, microcontroller for parking and rfid tags \& receivers are used for location estimation instead of using the traditional GPS method which has less resolution.
\end{abstract}

Keywords : Atmega162,IR Sensor,RFIDTags,Receivers,IOT

\section{INTRODUCTION}

Transportation is the major task in everyone's daily life whether it's for official reasons or for some personal reasons. Population increases in drastic manner and transportation also increased hugely. Further the number of vehicles usage also increases day by day. Development in economic status induces more usage of vehicles.There is no surprise in increasing usage of vehicles at developing countries like India in day to day life. Automobile industrial growth statistics gives proof for the above statement. Every household have at least one vehicle.Managing the vehicles in parking places becomes important in this modern busy lifestyle. Parking vehicles in an area also becomes complex nowadays. Manual and automatic modes are there in parking. While most of the places still uses manual mode in India. The types of parking also vary from place to place like open parking or underground parking.

Today most of the peoples are connected with the internet world and digital technology. Most of the problems are solved by simply sitting in one place in a secured way for e.g. financial transaction. This helps in efficient time usage with satisfied manner. With the embedded technology and digital communication problems in parking and retrieving he vehicles can be solved easily in a time efficient and secured way by the user itself.

\section{LITERATURE SURVEY}

In existing systems GPS and sensors are used to locate the vehicle and get the free slots in parking area respectively and the informationgiven to the user.In another way of

Revised Manuscript Received on August 19, 2019.

Francis Brindha, PG Student, Department of Electrical and Electronics Engineering, Kumaraguru College of Technology, Coimbatore.T.N, India (E-mail: francisbrindha.17mes@kct.ac.in)

A, Sandhya Devi RS, Assistant Professor, Department of Electrical and Electronics Engineering, Kumaraguru College of Technology, Coimbatore.T.N, India(E-mail: sandhyadevi.rs.eee@kct.ac.in) approach GPS and GSM is used to track the vehicle in real time and send the information to the user using SIM card respectively[3]. Automatic car parking system are available where the system will be parked automatically.In related work to this area sensor are used to locate the free parking slot and RFID tags are used to register the vehicle and to collect the parking fees[2].But using the GPS module one can't get the exact location of the device because of its less resolution and it also cant able to locate the device if it's a multifloor parking model.

\section{METHODOLOGY}

In this methodology of parking and tracking vehicles ,the information is obtained by using IR sensor ,RFID tag and RFID reader.IR sensors are placed in slots in the parking area and it indicates the user about the free and parked slots. RFID tag is placed in the vehicle (nowadays most of the car has RFID tag itself). RFID Reader is in movement inside the parking area. RFID Reader position is combined with slot area and its number. Whenever the user give request about its vehicle location inmobile app, the RFID reader gives the respective slot area to the user, in which place it reads the requested tag place [4]. RFID information and IR sensor information is controlled with the help of Microcontroller. Here Atmega162 is used. From this microcontroller information is sent tocloud with Wi-Fi module. All the information about free slot and parked slot is processed with the help of Wi-Fi and IoT cloud in a authenticated way using XMPP [1][2]. (Extensible Messaging and Presence Protocol)

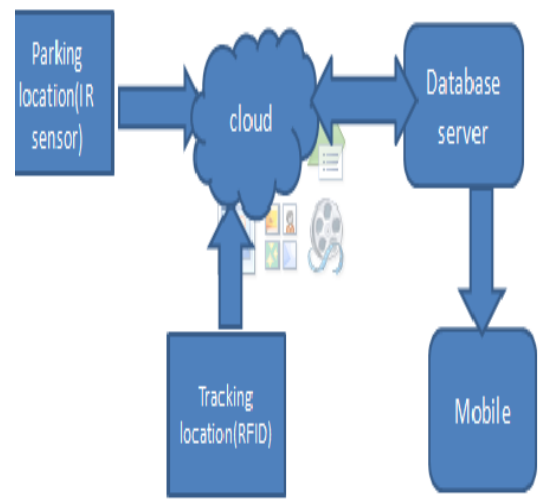

Fig. 1. Block Diagram 
Block diagram for this system is shown above. When the free slot is available after registering the vehicle in the parking area, car is parked. To locate the vehicle, after verifying the registration the location will be send to the user. Flow of this system is shown in Fig 2.

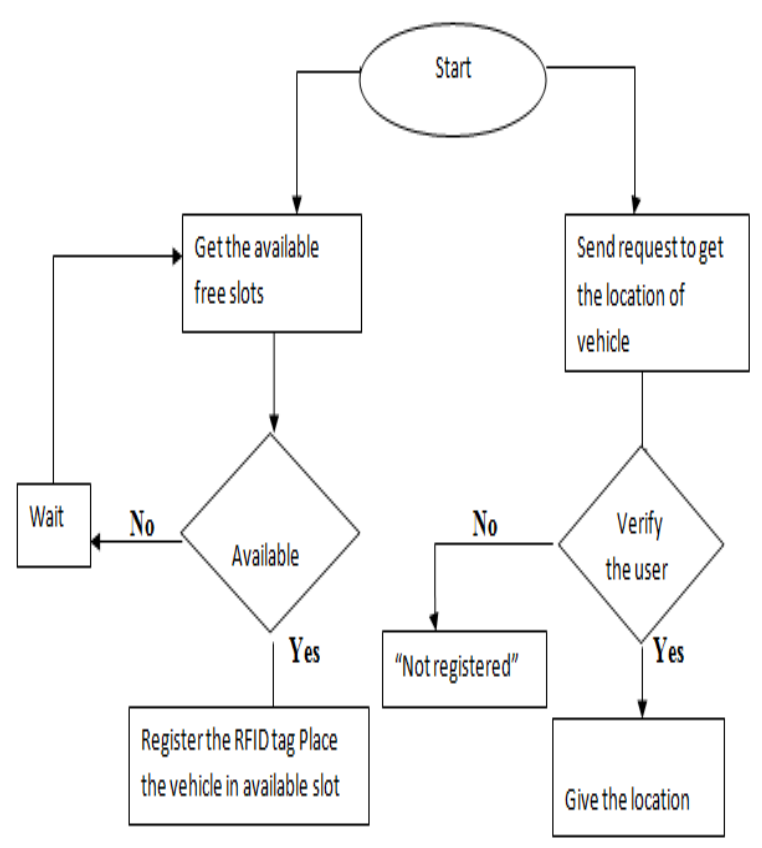

Fig. 2. Flow chart

The parking and tracking information will be sent to the cloud using Wi-Fi in a authenticated way. For this Authentication XMPP protocol is used, so that no third party can get the information.[7]

\section{IMPLEMENTATION}

\section{A.Parking Module}

IR sensors are used to locate the free slots in the parking area.IR sensors are places in the slots.If any car is there in slot, it will it and send the information as the car is detected otherwise send as slot is free.Flow chart is shown here.

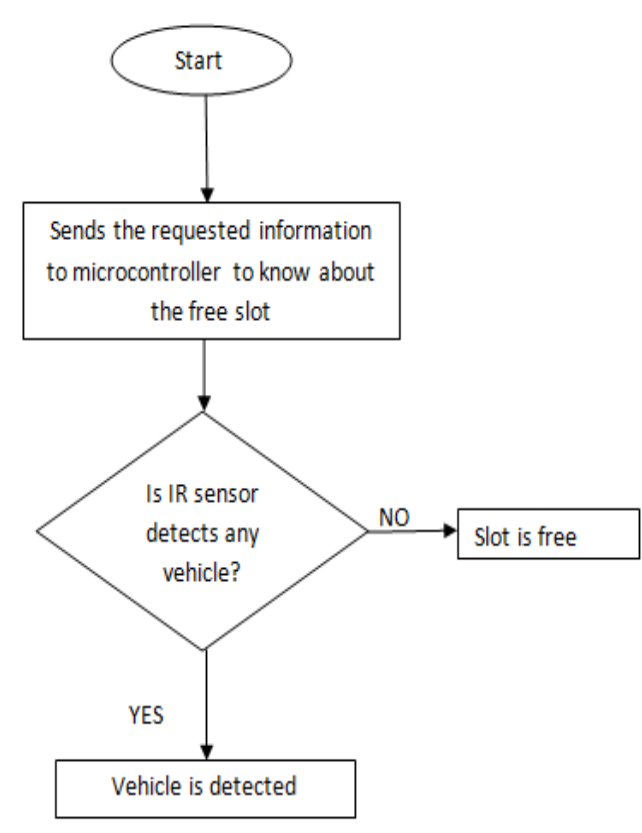

Fig. 3.Parking module

\section{B. Location Tracking Module}

RFID tags are placed inside the vehicle. RFID reader reads the tag while moving. When the RFID reader moving position is combined with slot area. Whenever the it reads the requested tag, it will send the information of the slot number which is already combined with it. By thisone can able to know the location of the vehicle. [5]

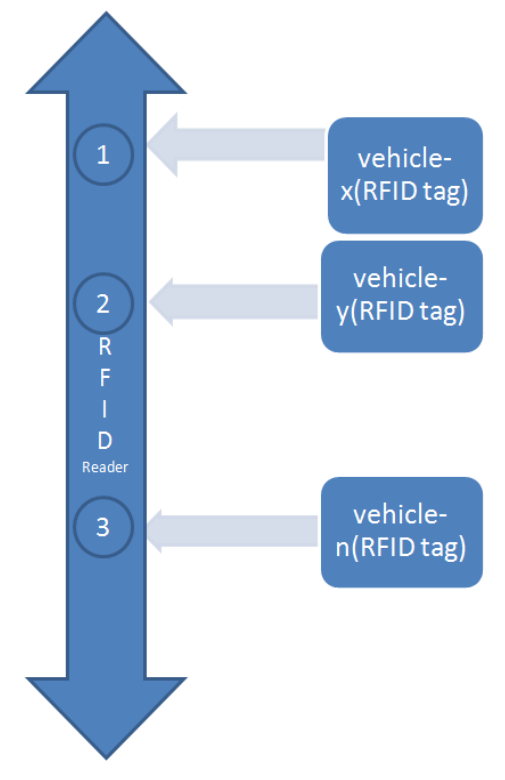

Fig.4. Tracking Module

\section{HARDWARE IMPLEMENTATION}

Images of the implemented module of getting the information about the slots using IR sensorin the LCD is shown below 


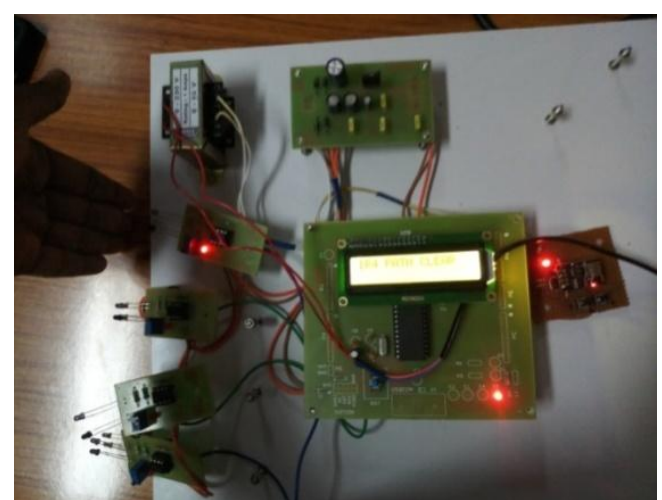

Fig. 5. Parking Module using IR sensor

Francis Brindha $\mathrm{A}$ is with Embedded System Technologies, KumaraguruCollege of Technology, Coimbatore, e-mail: francisbrindha.17mes@jct.ac.in.Sandhiya Devi RS is with EEE, Kumaraguru College of Technology, Coimbat- ore, email: sandhiya devi.rs.eee@kct.ac.in

\section{RESULTS AND DISCUSSION}

Using IR sensor free slot is identified and using RFID Tag and Reader is used to locate the vehicle. This information is displayed in the LCD now. In future this information will be send to cloud with Wi-Fi module and mobile app will be created to get the information for the user in a secured way by implementing XMPP protocol with the help of IoT.

\section{REFERENCES}

1. Antonio Celesti, Maria Fazio, Massimo Villari, "Enabling Secure XMPP Communications in Federated IoT Clouds Through XEP 0027 and SAML/SASL SSO”, journal Sensors 2017, 17, 301; doi:10.3390/s17020301.

2. Aniket Gupta, SujataKulkarni, VaibhaviJathar, Ved Sharma, Naman Jain, "Smart Car Parking Management System Using IoT", American Journal of Science, Engineering and Technology. Vol. 2, No. 4, 2017, pp. 112-119. doi: 10.11648/j.ajset.20170204.13.

3. HumaidAlshamsi, VetonKëpuska, HazzaAlshamsi, "Real Time Vehicle Tracking Using Arduino Mega", international Journal of Science and Technology (IJST) - Volume 5 No.12, December, 2016.

4. Balakrishnan, Nagaraj, ArunkumarRajendran, and KarthigaikumarPalanivel. "Meticulous fuzzy convolution C means for optimized big data analytics: adaptation towards deep learning." International Journal of Machine Learning and Cybernetics (2019): 1-12.

5. Ala Al-Fuqaha, Mohsen Guizani, Mehdi Mohammadi ,Mohammed Aledhari, MoussaAyyash, "Internet of Things: A Survey on Enabling Technologies, Protocols, and Applications", IEEE Communication Surveys \& Tutorials, Vol. 17, No. 4, Fourth Quarter 2015.

6. Reza Malekian Sensors (Basel),JacoPrinsloo "Accurate Vehicle Location System Using RFID, an Internet of Things Approach" 2016 Jun; 16(6): 825. Published online 2016 June.

7. SambandhBhusan Dhal, ArunAgarwal, and KabitaAgarwal, "Principles of Smart Car Parking Management System (With Efficient Corridor Lighting)." American Journal of Electrical and Electronic Engineering, vol. 4, no. 6 (2016): 152-156. doi: 10.12691/ajeee-4-6-1.

8. Hina C. Parmar, Nisha N. Shirv ,"Development of an Android Application for Smart Parking System," International Journal of Engineering Development and Research, (C) IJEDR 2018 Volume 6, Issue 2 | ISSN: 2321-9939. 